\title{
Gauge hierarchy problem and scalegenesis
}

\author{
Masatoshi Yamada* \\ Institut für Theoretische Physik, Universität Heidelberg, Philosophenweg 16, 69120 Heidelberg, \\ Germany \\ E-mail: m.yamada@thphys.uni-heidelberg.de
}

\begin{abstract}
We review the gauge hierarchy problem in the standard model. We discuss the meaning of the quadratic divergence in terms of the Wilsonian renormalization group. Classical scale symmetry, which prohibits dimensionful parameters in the bare action, could play a key role for the understanding of the origin of the electroweak scale. We discuss the scale-generation mechanism, i.e. scalegenesis in scale invariant theories. In this paper, we introduce a scale invariant extension of the SM based on a strongly interacting scalar-gauge theory. It is discussed that asymptotically safe quantum gravity provides a hint about solutions to the gauge hierarchy problem.
\end{abstract}

Corfu Summer Institute 2019 "School and Workshops on Elementary Particle Physics and Gravity" (CORFU2019)

31 August - 25 September 2019

Corfù, Greece

${ }^{*}$ Speaker. 


\section{Introduction}

The gauge hierarchy problem $[1,2]$ has been a long standing problem in the standard model (SM). The discovery of the Higgs boson with a mass of $125 \mathrm{GeV}[3,4]$, together with the lack of signals of new physics, implies that the SM could be valid up to a high scale. In particular, the renormalization group $(\mathrm{RG})$ analysis indicates that there is no obstacle to prevent the validity of the SM up to the Planck scale. This situation makes the gauge hierarchy problem more mysterious.

One of aspects of the gauge hierarchy problem relies on the interpretation of the quadratic divergence. Quantum corrections to the Higgs mass parameter contain both the logarithmic and quadratic divergences when employing a naive momentum cutoff or the Pauli-Villars regularization. The former divergence is proportional to the bare mass parameter, while the latter one is independent from it. The appearance of the quadratic divergence, however, strongly depends on a regularization scheme. Indeed, the dimensional regularization does not yield a corresponding object to the quadratic divergence. In the sense that physics should not depend on a choice of the regularization scheme, the quadratic divergence may be spurious.

The remaining gauge hierarchy problem after removing the quadratic divergence arises from the fact that the rapid energy scaling of the Higgs mass parameter: The dimensionless mass parameter behaves as $\bar{m}_{H}^{2}(k) \sim k^{-2}$, where $k$ is an energy scale. This implies that the ratio between the Higgs mass parameter and the Planck scale has to be $m_{H}^{2}\left(M_{\mathrm{pl}}\right) / M_{\mathrm{pl}}^{2} \sim 10^{-36}$ in order to realize $m_{H}^{2}\left(v_{h}\right) / v_{h}^{2} \sim 1$ at the electroweak scale. Why is the Higgs mass at the Planck scale so much smaller than the Planck scale? This question originates from the canonical dimension 2 of the scalar mass parameter. Although quantum effects induce an anomalous dimension $\gamma_{m}$ which deviates the energy scaling from the canonical one such that $2-\gamma_{m}$, it is negligibly small in the SM. A possible solution to this problem is the imposition of classical scale symmetry which prohibits dimensionful parameters in the bare action [5,6]. Then, there is no corresponding scale to the electroweak one, so that we need a scale-generation mechanism, i.e. scalegenesis. One of possible ways is the Coleman-Weinberg mechanism [7] in which scale symmetry is broken by the scale anomaly. The other scenario for scalegenesis relies on the strong dynamics like quantum chromodynamics. In both cases, a degree of freedom of a dimensionless coupling changes to that of a dimensionful parameter. This is the so-called dimensional transmutation.

The scale invariant SM, however, cannot realize electroweak scalegenesis, so that a scale invariant extension of the SM is required. The simplest extension is an introduction of a scalar field coupled to the Higgs field via the Higgs-portal coupling. If the dynamics in the new (hidden) sector generates a $\mathrm{TeV}$ scale, the electroweak symmetry breaking is triggered through the Higgs-portal coupling. It has been suggested a lot of possible scale invariant extensions as a hidden sector, together with other issues in the SM such as dark matter, neutrino masses and Baryogenesis.

However, classical scale invariance is nothing but a strong assumption. The clarification of the origin of classical scale invariance requires discussing the high energy physics including quantum gravity beyond the Planck scale. In this paper, we focus on the asymptotic safety scenario of quantum gravity. Asymptotically safe quantum gravity is formulated as a non-perturbative quantum field theory. An advantage of this scenario is that one can evaluate quantum gravity effects within the quantum field theory realm. With helps of the Wilsonian RG, we can calculate a large anomalous dimensions for a scalar mass parameter, $\gamma_{m}$ induced by graviton fluctuations. If $\gamma_{m}$ becomes 
larger than the canonical dimension 2, the energy scaling behavior of the scalar mass parameter is drastically changed. In such a scenario, we could have hints towards the gauge hierarchy problem.

This paper is organized as follows: In Section 2, we discuss renormalization using a simple scalar field theory. In particular, we explain the meaning of the quadratic divergence in viewpoint from the Wilson RG. Then, we discuss the gauge hierarchy problem and the idea of classical scale invariance in Section 3. Section 4 is devoted to introducing a mechanism for scalegenesis and to showing an example of an extension of the SM. In Section 5, we discuss impacts of graviton fluctuations in asymptotically safe quantum gravity on the scalar mass parameter, especially the anomalous dimension $\gamma_{m}$ induced by graviton fluctuations, and potential scenarios as a solution to the gauge hierarchy problem.

\section{Scalar mass and quadratic divergence in scalar theory}

In this section we discuss renormalization for a simple real singlet-scalar field theory. We start by reviewing the mass-independent renormalization (MIR) in the standard perturbation theory. It is shown in Section 2.1 that the scalar mass parameter is separated into the logarithmic and quadratic divergent parts. One can deal "multicatively" with the logarithmic divergence in renormalization, while the quadratic one is "additively" renormalized. In Section 2.2 we discuss the Wilson RG procedure for the same scalar theory. In particular, we highlight that the quadratic divergence in terms of the Wilsonian RG specifies an "absolute" position of the phase boundary between the broken and symmetric phases in the theory space for a chosen renormalization scheme. The independence of physical quantities from the choice of the renormalization scheme requires that they are defined as a deviation (relative distance) from the phase boundary. This can be actually seen by analyzing the RG flow around a fixed point. Then, the quadratic divergence becomes invisible by rotating the coordinate of the theory space around the fixed point.

\subsection{Mass-independent renormalization}

We demonstrate the MIR scheme using a real singlet-scalar theory whose Lagrangian reads

$$
\mathscr{L}=\frac{1}{2}\left(\partial_{\mu} \phi_{0}\right)^{2}-\frac{1}{2} m_{0}^{2} \phi_{0}^{2}-\frac{1}{4 !} \lambda_{0} \phi_{0}^{4}
$$

where the subscript " 0 " denotes a bare quantity. A key treatment of the MIR is that we split the bare mass into two parts, i.e. $m_{0}^{2}=\widehat{m}_{0}^{2}+\delta m_{0}^{2}$, and define renormalized quantities such that

$$
\phi=Z_{\phi}^{1 / 2} \phi_{0}, \quad m^{2}=Z_{m}^{-1} \widehat{m}_{0}^{2}, \quad \delta m^{2}=Z_{\phi} \delta m_{0}^{2}, \quad \lambda=Z_{\lambda}^{-1} \lambda_{0} .
$$

We should note here that the renormalized mass $m^{2}$ is proportional to $\widehat{m}_{0}^{2}$ but not to $\delta m_{0}^{2}$. For this setup the Lagrangian with counter terms in terms of the renormalized quantities (2.2) is given by

$$
\mathscr{L}=\frac{1}{2}\left(\partial_{\mu} \phi\right)^{2}-\frac{1}{2} m^{2} \phi^{2}-\frac{1}{4 !} \lambda \phi^{4}+\mathscr{L}_{\text {c.t. }},
$$

where the counter-term Lagrangian reads

$$
\mathscr{L}_{\text {c.t. }}=\frac{\delta Z_{\phi}}{2}\left(\partial_{\mu} \phi\right)^{2}-\frac{\delta Z_{m}}{2} m^{2} \phi^{2}-\frac{1}{2} \delta m^{2} \phi^{2}-\frac{\delta Z_{\lambda}}{4 !} \lambda \phi^{4},
$$


with $\delta Z_{\phi}=Z_{\phi}-1, \delta Z_{m}=Z_{m} Z_{\phi}-1$ and $\delta Z_{\lambda}=Z_{\lambda} Z_{\phi}^{2}-1$. To determine each counter term we employ the following four renormalization conditions:

$$
\begin{aligned}
& \left.\Gamma^{(2)}\left(p^{2}=0, m^{2}, \lambda ; \mu^{2}\right)\right|_{m^{2}=0}=0, \\
& \left.\frac{\partial}{\partial m^{2}} \Gamma^{(2)}\left(p^{2}=0, m^{2}, \lambda ; \mu^{2}\right)\right|_{m^{2}=\mu^{2}}=-1, \\
& \left.\frac{\partial}{\partial p^{2}} \Gamma^{(2)}\left(p^{2}, m^{2}, \lambda ; \mu^{2}\right)\right|_{p^{2}=0, m^{2}=\mu^{2}}=1, \\
& \left.\Gamma^{(4)}\left(\left\{p_{i}\right\}=0, m^{2}, \lambda ; \mu^{2}\right)\right|_{m^{2}=\mu^{2}}=-\lambda,
\end{aligned}
$$

where $\mu$ is the renormalization scale; $\Gamma^{(2)}$ and $\Gamma^{(4)}$ stand for the two- and four-point functions of the scalar field, respectively; $p$ is an external momentum; and $\left\{p_{i}\right\}$ denotes a set of external momenta, e.g. $\left\{p_{i}\right\}=\left(p_{1}, p_{2}, p_{3}, p_{4}\right)$ for the four-point function. ${ }^{1}$

Let us now calculate the two-point function. At the one-loop level, one can obtain

$$
\begin{aligned}
\left.\Gamma^{(2)}\left(p^{2}\right)\right|_{\mathscr{O}\left(\lambda^{1}\right)} & = \\
& =-\frac{i \lambda}{2} \int \frac{\mathrm{d}^{4} q}{(2 \pi)^{4}} \frac{1}{q^{2}-m^{2}}+\delta Z_{\phi}^{(1)} p^{2}-\delta Z_{m}^{(1)} m^{2}-\left(\delta m^{2}\right)^{(1)}
\end{aligned}
$$

Here the one-loop integration is calculated as

$$
\begin{aligned}
& -\frac{i \lambda}{2} \int \frac{\mathrm{d}^{4} q}{(2 \pi)^{4}} \frac{1}{q^{2}-m^{2}} \\
& =\left\{\begin{array}{cc}
-\frac{\lambda}{32 \pi^{2}}(2 \ln 2) \Lambda^{2}+\frac{\lambda}{32 \pi^{2}} m^{2}\left(1+\ln \frac{\Lambda^{2}}{m^{2}}\right) & \text { (Pauli-Villars reg.) } \\
+\frac{\lambda}{32 \pi^{2}} m^{2}\left(1+\frac{1}{\varepsilon}\right) & \text { (dimensional reg.) }
\end{array},\right.
\end{aligned}
$$

where $\Lambda$ is a UV cutoff in the Pauli-Villars regularization, ${ }^{2}$ and we defined $1 / \varepsilon=2 /(4-d)-\gamma_{E}+$ $\ln (4 \pi)$ with $\gamma_{E}$ the Euler gamma constant and $d$ spacetime dimension. From the conditions (2.6) and (2.7), we can determine the field and mass renormalization factors,

$$
\delta Z_{\phi}^{(1)}=0, \quad \delta Z_{m}^{(1)}=\frac{\lambda}{32 \pi^{2}}\left(1+\left\{\ln \frac{\Lambda^{2}}{\mu^{2}} \text { or } \frac{1}{\varepsilon}\right\}\right)
$$

\footnotetext{
${ }^{1}$ We note that one of momenta is redundant due to the momentum conservation.

${ }^{2} \mathrm{To}$ regularize the UV divergence, we employ the following replacement for the scalar propagator:

$$
\frac{1}{q^{2}-m^{2}} \rightarrow\left(\frac{1}{q^{2}-m^{2}}-\frac{1}{q^{2}-\Lambda^{2}}\right)-\left(\frac{1}{q^{2}-\left(\Lambda^{2}+m^{2}\right)}-\frac{1}{q^{2}-2 \Lambda^{2}}\right) \text {. }
$$


while the first condition (2.5) gives

$$
\left(\delta m^{2}\right)^{(1)}=\left\{\begin{array}{lc}
-\frac{\lambda}{32 \pi^{2}}(2 \ln 2) \Lambda^{2} & (\text { Pauli-Villars reg. }) \\
0 & \text { (dimensional reg. })
\end{array}\right.
$$

We see that $\delta m^{2}$ subtracts the quadratic divergence $\Lambda^{2}$ in the Pauli-Villars regularization. Thus, the MIR scheme allows us to separate the bare mass into the logarithmically divergent part and the quadratically divergent part. In the dimensional regularization the quadratic divergence is automatically subtracted without the introduction of $\delta m^{2}$. Note here that $Z_{\phi}$ and $Z_{m}$ do not depend on the renormalized mass parameter $m^{2}$. Indeed, $Z_{\lambda}$ is also independent from $m^{2}$. This is why this renormalization scheme is called the "mass-independent" renormalization. Note also that it is guaranteed in a fermionic theory that the bare fermion mass term $\delta m_{0}$ is zero in any regularization scheme thanks to chiral symmetry, so that the bare mass is always proportional to the renormalized mass. In a gauge theory, the gauge symmetry realizes the same situation.

Once one obtains the renormalization factors, one can evaluate the anomalous dimensions which characterize the energy scaling of coupling constants,

$$
\beta(\lambda)=-\lambda \mu \frac{\partial \ln Z_{\lambda}}{\partial \mu}, \quad \gamma_{m}(\lambda)=-\mu \frac{\partial \ln Z_{m}}{\partial \mu} . \quad \gamma_{\phi}(\lambda)=\frac{1}{2} \mu \frac{\partial \ln Z_{\phi}}{\partial \mu} .
$$

From the second definition in Eq. (2.14) and the definition of the renormalized scalar mass, one obtains

$$
\mu \frac{\partial}{\partial \mu} \bar{m}^{2}=\left(-2+\gamma_{m}\right) \bar{m}^{2}
$$

where $\bar{m}^{2}=m^{2} / \mu^{2}$ is the dimensionless scalar mass, and the anomalous dimension at the one-loop level reads

$$
\gamma_{m}=\frac{1}{16 \pi^{2}} \lambda
$$

Since $Z_{\phi}=1$ at the one-loop level, one has $\gamma_{\phi}=0$. The RG equation for the quartic coupling constant at the one-loop level is well known as

$$
\mu \frac{\partial}{\partial \mu} \lambda=\frac{3 \lambda^{2}}{16 \pi^{2}}
$$

The solution to this equation behaves $\lambda \rightarrow 0$ for $\mu \rightarrow 0$, while there is a Landau pole at $\mu=\mu_{0} e^{\frac{16 \pi^{2}}{3 \lambda\left(\mu_{0}\right)}}$ where $\lambda\left(\mu_{0}\right)$ is a boundary value at $\mu=\mu_{0}$.

\subsection{Wilsonian renormalization group}

Let us briefly introduce the Wilsonian RG in quantum field theory. The task in quantum field theory is to solve the path integral which represents the summation of all quantum fluctuations. We now consider to divide quantum fluctuations into high momentum modes and low momentum modes by introducing an infrared (IR) cutoff scale $k$ such that $\phi(p)=\phi_{>}(p)+\phi_{<}(p)$ where $\phi_{>}(p)$ 
and $\phi_{<}(p)$ are fields with higher momentum modes $|p|>k$, and lower momentum modes $|p|>k$, respectively. Thus, we perform the path integral only for the field $\phi_{>}(p)$ and define an effective action which is a functions of $\phi_{<}(p)$. This operation is the so-called coarse-graining. Varying the IR cutoff scale to $k=0$, we can obtain the full effective action including all quantum fluctuations. Such a process can be formulated as a functional differential equation. One of formulations is given for the one-particle irreducible (1PI) effective action $\Gamma_{k}[8]$ so that

$$
\partial_{t} \Gamma_{k}=\frac{1}{2} \operatorname{Tr}\left[\left(\Gamma_{k}^{(2)}+R_{k}\right)^{-1} \cdot \partial_{t} R_{k}\right],
$$

where $R_{k}(p)$ is a cutoff profile function (or simply a regulator) realizing the coarse-graining, $\Gamma_{k}^{(2)}$ is the full two-point function, namely $\Gamma_{k}^{(2)}=\frac{\delta^{2}}{\delta \phi^{2}} \Gamma_{k}$, and " $\operatorname{Tr}$ " denotes the functional trace acting on all internal spaces in which the field is defined, e.g. momentum and flavor, etc.

The 1PI effective effective action is in general spanned by an infinite number of effective operators $\mathscr{O}_{i}(x)$ :

$$
\Gamma_{k}=\int \mathrm{d}^{4} x \sum_{i}^{\infty} g_{i} \mathscr{O}_{i}(x),
$$

where $g_{i}$ is a dimensionful coupling constant. Using the flow equation (2.18) and defining a dimensionless coupling constant $\bar{g}_{i}=k^{-\left(4-D_{i}\right)} g_{i}$, where $D_{i}$ is the mass dimension of $\mathscr{O}_{i}$, one obtains the RG equation for $\bar{g}_{i}$,

$$
\partial_{t} \bar{g}_{i}=\beta_{i}(\{\bar{g}\}),
$$

where $\{\bar{g}\}$ denotes a set of coupling constants. The right-hand side of Eq. (2.20) is the beta function.

One of important things in the RG is the existence of a fixed point $\bar{g}_{i *}$ at which all beta functions vanish, i.e. $\beta_{i}\left(\left\{\bar{g}_{*}\right\}\right)=0$ for all $i$. Once a fixed point is found, one can analyze the RG flows around the fixed point. To this end, we expand the beta functions in Eq. (2.20) around a fixed point $g_{i *}$ and take into account only the linear term,

$$
\partial_{t}\left(\bar{g}_{i}-\bar{g}_{i}^{*}\right) \simeq-T_{i j}\left(\bar{g}_{j}-\bar{g}_{j *}\right),
$$

where we have defined the stability matrix,

$$
T_{i j}=-\left.\frac{\partial \beta_{i}}{\partial \bar{g}_{j}}\right|_{g=g_{*}} .
$$

The equation (2.21) can be solved easily: Diagonalizing the stability matrix (2.22) and denoting its eigenvalues by $\theta_{i}$, one finds

$$
\bar{g}_{i}-\bar{g}_{i *}=\sum_{j} V_{i j} C_{j}\left(\frac{k}{\Lambda}\right)^{-\theta_{j}} .
$$

Here, $V_{i j}$ is a matrix diagonalizing the stability matrix (2.22) and $C_{j}$ is a constant at a reference scale $\Lambda$. When the matrix $V$ is approximated to be diagonal, $V_{i j} \approx v_{i} \delta_{i j}$, one has

$$
\bar{g}_{i}-\bar{g}_{i *}=v_{i} C_{i}\left(\frac{k}{\Lambda}\right)^{-\theta_{i}}
$$


One can see from Eq. (2.24) that $\theta_{i}$ characterizes the energy scaling of coupling constant $\bar{g}_{i}$ and is called as the "critical exponent". In particular, for a found fixed point, the sign of the critical exponents is important. There are the following cases:

$$
\begin{array}{ll}
\theta_{i}>0 & \text { relevant } \\
\theta_{i}=0 & \text { marginal } \\
\theta_{i}<0 & \text { irrelevant }
\end{array}
$$

Towards IR regimes, the RG flow of a relevant coupling $\left(\theta_{i}>0\right)$ goes away from the fixed point, while that of an irrelevant one $\left(\theta_{i}<0\right)$ shrinks to the fixed point. This fact leads to the notion of the renomalizability of a theory. Since irrelevant couplings, starting at an arbitrary value in a UV scale, converge to the fixed point value in the low energy region, they are predictable, i.e. $\bar{g}_{i}=\bar{g}_{i *}$. If a theory has a finite number of relevant couplings, the theory is renormalizable.

A typical structure of the beta functions becomes the following form:

$$
\partial_{t} \bar{g}_{i}=-\left(4-D_{i}\right) \bar{g}_{i}+f_{i}(\{\bar{g}\}),
$$

where $D_{i}$ is the mass-dimension of a corresponding operator to $g_{i}$. The first term on the right-hand side is the canonical scaling term reflecting the mass-dimensionality of the coupling $\bar{g}_{i}$, and the second one involves quantum effects. Let us here assume that the system described by Eq. (2.26) has a fixed point $\bar{g}_{i *}$. In this case, the stability matrix is given by

$$
T_{i j}=\left(4-D_{i}\right) \delta_{i j}+\left.\frac{\partial f_{i}}{\partial \bar{g}_{j}}\right|_{g=g_{*}},
$$

from which the critical exponent is obtained as $\theta_{i}=\operatorname{eig}_{i}(T)$.

In particular, for the Gaussian fixed point $\bar{g}_{i *}=0$ or a small one $\bar{g}_{i *} \ll 1$, the quantum effects (second term on the right-hand side in Eq. (2.27)) are negligible, so that the critical exponent is identical with the canonical scaling, i.e. $\theta_{i}=4-D_{i}$. This is consistent with the standard definition of the renormalizability in the perturbation theory. One can see the critical exponents at the Gaussian fixed point from a naive dimensional counting of coupling constants, whereas at a nonvanishing fixed point, in general, the second term on the right-hand side in Eq. (2.27) is finite, so that the critical exponents deviate from the canonical scalings of coupling constants, i.e. the second term is the anomalous dimension induced by quantum effects.

We now analyze a scalar theory using the flow equation (2.18). Quantum effects generate an infinite number of effective operators which respect with symmetries the bare action has. We assume that the scalar theory has the $Z_{2}$ symmetry which admits only even powers of $\phi$ in the effective action. For the present purpose we take only the following three terms into account and truncate higher operators:

$$
\Gamma_{k}[\phi] \simeq \int \mathrm{d}^{4} x\left[\frac{Z_{\phi}^{-1}}{2}\left(\partial_{\mu} \phi\right)^{2}+\frac{1}{2} m^{2} \phi^{2}+\frac{1}{4 !} \lambda \phi^{4}\right]
$$


where we consider the Euclidean spacetime. Using the flow equation (2.18) one obtains

$$
\begin{aligned}
\partial_{t} \bar{m}^{2} & =-2\left(1-\gamma_{\phi}\right) \bar{m}^{2}-\frac{\bar{\lambda}}{32 \pi^{2}} \ell_{1}^{4}\left(\bar{m}^{2}\right), \\
\partial_{t} \bar{\lambda} & =4 \gamma_{\phi} \bar{\lambda}+\frac{3 \bar{\lambda}^{2}}{16 \pi^{2}} \ell_{2}^{4}\left(\bar{m}^{2}\right),
\end{aligned}
$$

where a bar denotes a dimensionless quantity, the first terms on the right-hand side of Eqs. (2.29) and (2.30) are canonical scaling terms which reflect the canonical dimensions of the scalar mass term and the quartic coupling, and the second terms are loop effects. See e.g. Ref. [9] for the derivation of these RG equations. Here, $\gamma_{\phi}=\partial_{t} \ln Z_{\phi} / 2$ is the anomalous dimension from the field renormalization factor and we define the threshold functions in four dimensional spacetime,

$$
\ell_{0}^{4}(\tilde{w})=k^{-2} \int_{0}^{\Lambda^{2}} \mathrm{~d} x \frac{x}{2} \frac{\partial_{t} R_{k}(x)}{x+R_{k}(x)+\tilde{w}}, \quad \ell_{n}^{4}(\tilde{w})=(-1)^{n} \frac{1}{n !} \frac{\partial^{n}}{\partial \tilde{w}} \ell_{0}^{4}(\tilde{w}) .
$$

For the Litim-type cutoff function [10],

$$
R_{k}\left(p^{2}\right)=\left(a k^{2}-p^{2}\right) \theta\left(a k^{2}-p^{2}\right)
$$

we can perform the momentum integral in the threshold function (2.31) and then obtain

$$
\ell_{n}^{4}\left(\bar{m}^{2}\right)=\frac{a^{-n+2}}{\left(1+a^{-1} \bar{m}^{2}\right)^{n+1}},
$$

where $a$ is a (dimensionless) positive and finite constant and describes a class of regularization schemes. Note here that $\ell_{2}^{4}(0)$ is independent from the parameter $a$.

Let us solve the RG equations (2.29) and (2.30). For simplicity, we assume that $a^{-1} \bar{m}^{2} \ll 1$, i.e. we can expand the threshold functions as

$$
\ell_{1}^{4}\left(\bar{m}^{2}\right) \approx \ell_{1}^{4}(0)-2 \ell_{2}^{4}(0) \bar{m}^{2}, \quad \quad \ell_{2}^{4}\left(\bar{m}^{2}\right) \approx \ell_{2}^{4}(0) .
$$

We neglect the anomalous dimension of the field renormalization factor, $\gamma_{\phi}=0$ (or equivalently $\left.Z_{\phi}^{-1}=1\right){ }^{3}$ Indeed, for scalar theories in the symmetric phase, the field renormalization factor does not deviate from one. Then, the RG equation for the scalar mass is simplified to be

$$
\partial_{t} \bar{m}^{2}=-2 \bar{m}^{2}-\frac{a \bar{\lambda}}{32 \pi^{2}}+\frac{\bar{\lambda}}{16 \pi^{2}} \bar{m}^{2}
$$

where we have used $\ell_{1}^{4}(0)=a$ and $\ell_{2}^{4}(0)=1$ in the Litim-type cutoff (2.32). Compare this to Eq. (2.15). The last term on the right-hand side of Eq. (2.35) can be identified to $\gamma_{m}$, while the second term is missing in Eq. (2.15). This term is actually the contribution from the quadratic divergence: By solving Eq. (2.35) without the last term of Eq. (2.35), one finds, for the dimensionful scalar mass,

$$
m^{2}(k \rightarrow 0)=-\frac{a \bar{\lambda}}{64 \pi^{2}} \Lambda^{2}
$$

\footnotetext{
${ }^{3}$ This approximation is called as the local potential approximation.
} 
The Wilsonian RG does not respect to scale symmetry due to the introduction of the cutoff scale $k$, so that in general, the quadratic divergence appears in the beta function of the scalar mass. The quadratic divergent part explicitly depends on the parameter $a$.

What is the meaning of the quadratic divergence in the Wilsonian RG? To answer to this question, let us analyze the RG flow around a fixed point in the scalar system. In a scalar theory in four dimensional spacetime, one could find, for a positive quartic coupling constant, ${ }^{4}$ only the Gaussian (trivial) fixed point, $\bar{m}_{*}^{2}=\bar{\lambda}_{*}=\cdots=0$ at which the linearized RG equation (2.21) reads

$$
\left.\partial_{t}\left(\begin{array}{c}
\bar{m}^{2} \\
\bar{\lambda}
\end{array}\right) \simeq\left(\begin{array}{cc}
\frac{\partial \beta_{m}}{\partial \bar{m}^{2}} & \frac{\partial \beta_{m}}{\partial \bar{\lambda}} \\
\frac{\partial \beta_{\lambda}}{\partial \bar{m}^{2}} & \frac{\partial \beta_{\lambda}}{\partial \bar{\lambda}}
\end{array}\right)\right|_{\substack{\bar{m}_{*}^{2}=0 \\
\bar{\lambda}_{*}=0}}\left(\begin{array}{c}
\bar{m}^{2} \\
\bar{\lambda}
\end{array}\right)=\left(\begin{array}{cc}
-2 & -\frac{a}{32 \pi^{2}} \\
0 & 0
\end{array}\right)\left(\begin{array}{c}
\bar{m}^{2} \\
\bar{\lambda}
\end{array}\right) .
$$

Note that the term $-\frac{a}{32 \pi^{2}}$ comes from the quadratically divergent part (the second term on the right-hand side) in Eq. (2.35). Diagonalizing the stability matrix by the rotation matrix,

$$
V=\left(\begin{array}{cc}
1 & -\frac{a}{64 \pi^{2}} \\
0 & 1
\end{array}\right),
$$

we find one of the linearized RG equations,

$$
\partial_{t}\left(\bar{m}^{2}+\frac{a}{64 \pi^{2}} \bar{\lambda}\right)=-2\left(\bar{m}^{2}+\frac{a}{64 \pi^{2}} \bar{\lambda}\right)
$$

and its solution,

$$
\bar{m}^{2}(k)+\frac{a}{64 \pi^{2}} \bar{\lambda}(k)=e^{2 t}\left(\bar{m}_{0}^{2}+\frac{a}{64 \pi^{2}} \bar{\lambda}_{0}\right)
$$

where $\bar{m}_{0}^{2}=\bar{m}^{2}(k=\Lambda)=m_{0}^{2} / \Lambda^{2}$ and $\bar{\lambda}_{0}=\bar{\lambda}(k=\Lambda)=\lambda_{0}$. Note that $k=\Lambda$ corresponds to $t=0$. We see that for the choice at the UV scale,

$$
m_{0}^{2}=-\frac{a \bar{\lambda}_{0}}{64 \pi^{2}} \Lambda^{2}
$$

the RG flow (2.40) gives the relation

$$
\bar{m}^{2}(k)=-\frac{a}{64 \pi^{2}} \bar{\lambda}(k)=: \bar{m}_{c}^{2}(\bar{\lambda}) .
$$

Since the RG flow of the quartic coupling constant goes to zero in the IR limit $k \rightarrow 0$, one can see $\bar{m}^{2}(k \rightarrow 0) \rightarrow 0$ from this equation (2.42). Hence, the choice (2.41) is a condition to obtain the massless theory at the IR limit and the relation (2.42) describes the RG trajectory towards the massless theory. It is clear from Eq. (2.40) that for a choice $m_{0}^{2}>\bar{m}_{c}^{2}\left(\bar{\lambda}_{0}\right)$, the scalar mass takes a positive value in the IR limit, namely the theory is in the symmetric phase in the IR regime. In the opposite inequality case $\left(m_{0}^{2}<\bar{m}_{c}^{2}\left(\bar{\lambda}_{0}\right)\right)$, the broken phase is observed in the IR regime. To summarize, the relation (2.42) represents the phase boundary (critical line) between the symmetric and broken phases for the $Z_{2}$ symmetry $[5,12]$. In particular, the theory on the phase boundary is massless, i.e. critical. In Fig. 1, we plot the RG flows in the $\bar{m}^{2}-\bar{\lambda}$ plane. The red line in Fig. 1 is the phase boundary around the Gaussian fixed point described by Eq. (2.42).

\footnotetext{
${ }^{4}$ If we extend the quartic coupling constant to negative values, there exists a non-trivial fixed point [11].
} 

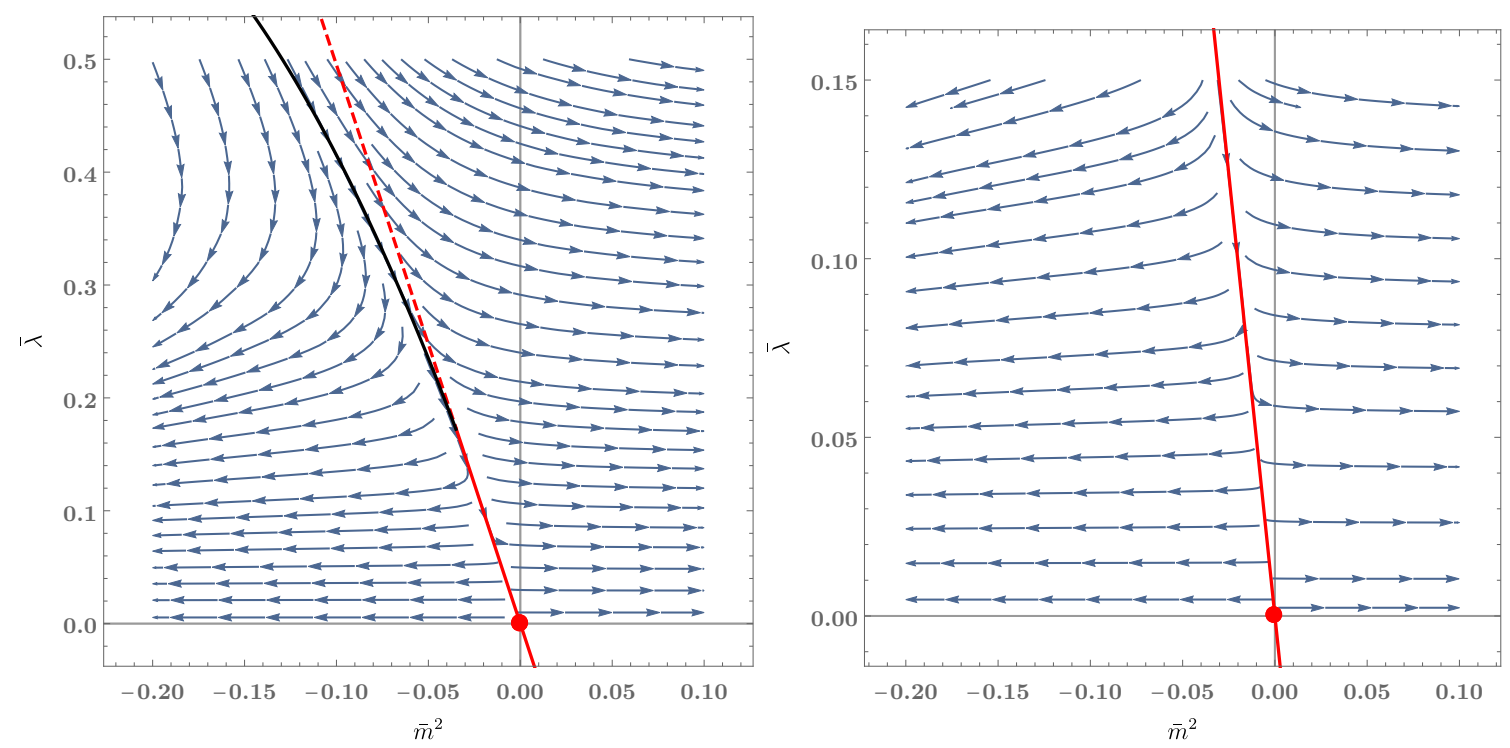

Figure 1: RG flows in the $\bar{m}^{2}-\bar{\lambda}$ plane. Arrows indicate flows from UV to IR. The red point is the Gaussian fixed point $\left(\bar{m}_{*}^{2}=0, \bar{\lambda}_{*}=0\right)$. The red and black solid lines denote the phase boundaries around and apart from the Gaussian fixed point, respectively.

However, the slope of this phase boundary strongly depends on the regularization scheme. Indeed, the choice of the regularization scheme corresponds to the choice of the coordinate in the theory space [13]. A choice of the coordinate is connected to other choices by rotations of the coordinate. Therefore, one can rotate arbitrary coordinates such that the quadratic divergence is subtracted. The matrix corresponding to such a rotation is actually given by Eq. (2.38) in the present analysis. In this sense, the dimensional regularization is a scheme so that the rotation matrix becomes the identity matrix.

The quantities after the rotation correspond to the relative distance between the RG flow and the phase boundary (or the deviation from the phase boundary) are universal, i.e. independent from the regularization scheme. From Eq. (2.40) one can define

$$
\widehat{m}_{0}^{2}=m_{0}^{2}+\frac{a \bar{\lambda}_{0}}{64 \pi^{2}} \Lambda^{2}, \quad \quad \widetilde{m}^{2} \equiv \bar{m}^{2}+\frac{a}{64 \pi^{2}} \bar{\lambda} .
$$

This is the scalar mass in the new basis for the theory space. The RG equations for the scalar mass and the quartic coupling constant in the new basis are given repectively by

$$
\partial_{t} \widetilde{m}^{2}=-2 \widetilde{m}^{2}, \quad \partial_{t} \bar{\lambda}=0 .
$$

The RG flows in the new basis is represented in Fig. 2. The one-loop effects in these equations are missing. To take into account them, we have to evaluate non-linear terms in Eq. (2.37). For a large value of the quartic coupling constant the phase boundary is bended as one can see from the black solid line in Fig. 1.

\section{Gauge hierarchy problem in the SM and classical scale invariance}

So far, we have discussed renormalization in a simple scalar theory for both the standard 


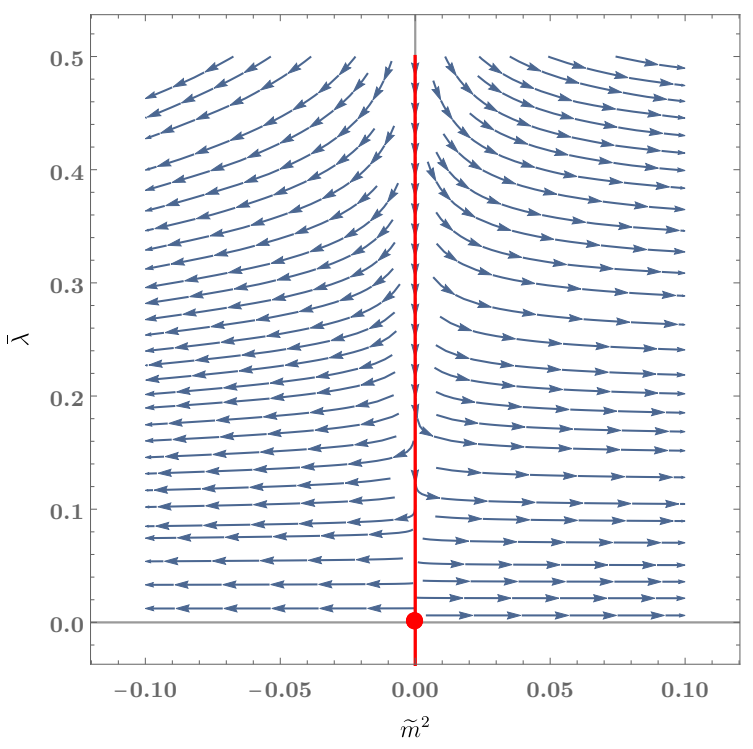

Figure 2: RG flows in the $\widetilde{m}^{2}-\bar{\lambda}$ plane, where $\widetilde{m}^{2}$ is defined in Eq. (2.43).

perturbative renormalization and the Wilsonian RG. In particular, we have argued the meaning of the quadratic divergence. In the SM with the momentum regularization, one finds, at the one-loop level, ${ }^{5}$

$$
\delta m^{2}=\frac{1}{16 \pi^{2}}\left(-6 \lambda+6 y_{t}^{2}-\frac{9}{4} g_{2}^{2}-\frac{3}{4} g_{1}^{2}\right) \Lambda^{2},
$$

where $\lambda$ is the quartic coupling constant of the Higgs doublet-field; $y_{t}$ is the top-quark Yukawa coupling constant; and $g_{2}$ and $g_{1}$ are the $\mathrm{SU}(2)_{L}$ and $\mathrm{U}(1)_{Y}$ gauge coupling constants, respectively. If the SM is valid up to the Planck scale, the quadratic divergence has to be precisely canceled with the bare mass $\delta m_{0}^{2}$ which does not depend on the renormalized scalar mass $m^{2}$. This is the so-called gauge hierarchy problem or fine-tuning problem $[1,2]$. This problem has motivated us to consider supersymmetric extensions of the SM, in which the quadratic divergences from a particle and its superpartner cancel each other out; see e.g. Ref. [15]. An other idea for a solution to the gauge hierarchy problem is the Veltman condition [16] which states that the coefficient of the quadratic divergence (the combination of coupling constants in Eq. (3.1)) should vanish at a UV scale at which the bare theory is defined.

Is the quadratic divergence, however, physically meaningful? As discussed in the previous section, the quadratic divergence is always subtracted by the counter term $\delta m^{2}$. In view point of the Wilsonian RG, the quadratic divergence specifies the position of the phase boundary and strongly depends on the choice of the coordinate (regularization scheme) in the theory space. Rotating the coordinate around the Gaussian fixed point, the quadratic divergence is invisible in the new basis of the coordinate. If high energy theories such as string theory determine the coordinate of the theory space, one has to seriously discuss the gauge hierarchy problem why the quadratic divergence is precisely canceled out with the bare Higgs mass. Nevertheless, as far as one discusses the dynamics

\footnotetext{
${ }^{5}$ The two-loop contribution has been computed, e.g. in Ref. [14].
} 
of particles within low energy effective theories, one does not have to specify the scheme-dependent coordinate of the theory space, so that the quadratic divergence may be spurious.

Once we accept the idea that the quadratic divergence is irrelevant for the low energy dynamics, we can consider only the logarithmic divergence which contributes to the running of the scalar mass. Is the gauge hierarchy problem resolved if the quadratic divergence is subtracted? Its answer is No. To see the remaining problem, let us study the RG equation given in Eq. (2.15) or Eq. (2.35) after the quadratic divergence is subtracted. Assuming that the running effects of the anomalous dimension $\gamma_{m}$ is negligible, the running (dimensionless) scalar mass parameter is given by

$$
\bar{m}^{2}(\mu)=\bar{m}_{0}^{2}\left(\frac{\mu}{\Lambda}\right)^{-2+\gamma_{m}}
$$

where $\bar{m}_{0}^{2}$ is the boundary mass at the UV scale, i.e. $\bar{m}_{0}^{2}=\bar{m}^{2}(\mu=\Lambda)=\widehat{m}_{0}^{2} / \Lambda^{2}$. In the SM, the anomalous dimension at one-loop level is computed as

$$
\gamma_{m}=\frac{1}{16 \pi^{2}}\left(2 \lambda+6 y_{t}^{2}-\frac{9}{2} g_{2}^{2}-\frac{3}{2} g_{1}^{2}\right)
$$

This value at the electroweak scale is $\gamma_{m} \approx 0.027$, so that it is negligible in comparison with the canonical scaling 2 in the RG equation for the scalar mass (2.15). We here evaluate the value of $\bar{m}_{0}$ when a bare theory of the SM is given at the Planck scale $\Lambda=M_{\mathrm{pl}} \simeq 10^{19} \mathrm{GeV}$ using Eq. (3.2) with $\gamma_{m}=0$. The observed Higgs boson mass $m_{H}=125 \mathrm{GeV}$ and the electroweak scale $v_{h}=246 \mathrm{GeV}$ tell us that the dimensionless renormalized mass at the electroweak scale is given as $\bar{m}^{2}\left(\mu=v_{h}\right)=$ $m_{H}^{2} / v_{h}^{2} \simeq 0.1$. From this one finds $\bar{m}^{2}\left(\mu=M_{\mathrm{pl}}\right)=m_{0}^{2} / M_{\mathrm{pl}}^{2} \simeq 10^{-36}$ at the Planck scale. Hence, the scalar mass in the bare action is quite smaller than the Planck scale at which the bare action is defined. This is the remaining gauge hierarchy problem.

This gauge hierarchy problem originates from the fact that the scalar mass term is a relevant parameter with the large critical exponent $\theta_{m} \approx 2$. Therefore, one of simple possible solutions to this problem is to have a large anomalous dimension $\gamma_{m}$ such that $\theta_{m}=2-\gamma_{m} \lesssim 0$; see e.g. [17, $18,20,19]$. To realize such a situation, we need strong dynamics. However, new particles strongly coupled to the Higgs field around the $\mathrm{TeV}$ scale are highly constrained by the collider experiments such as the LHC.

An other possibility is classical scale invariance [5, 6]. Classical scale symmetry forbids the renormalized dimensionful mass $m^{2}$ at the UV scale $\Lambda$. Hence it enforces, for the bare theory,

$$
\widehat{m}_{0}^{2}=0
$$

In this case, the renormalized scalar mass keeps vanishing under varying the RG scale as can be seen in Eq. (3.2). This fact means that the breaking of scale symmetry due to the scale anomaly is not soft, but is hard. On the other hand, how does classical scale symmetry act on $\delta m_{0}^{2}$ which is also a dimensionful parameter? Scale symmetry is broken by the quadratic divergence coming from regularizations such as the Pauli-Villars regularization and the momentum regularization. Such an explicit breaking should be modified in accordance with the Ward-Takahashi identity for scale symmetry, i.e. the Callan-Symanzik equation [21, 22]. Hence, one can interpret $\delta m_{0}^{2}$ as an additive counter term in order to remove the spurious breaking of scale symmetry. Needless to 
say, the dimensional regularization respects to scale symmetry and then yields $\delta m_{0}^{2}=0$.Then the massless scalar theory is renormalizable [23].

In the viewpoint from the Wilsonian renormalization group, the massless theory is realized at the phase boundary (critical line). The quite small Higgs mass $\widehat{m}_{0} / M_{\mathrm{pl}} \simeq 10^{-36}$ means that the bare Higgs is located very near the phase boundary. In this sense, the gauge hierarchy problem can be paraphrased as the criticality problem: Why is the Higgs so close to critical? Classical scale symmetry forces the Higgs to be put exactly on the phase boundary: Classical scale symmetry makes the Higgs critical.

The theory with classical scale invariance has no corresponding scale to the electroweak one, so that one needs to a mechanism in order to generate a scale, i.e. scalagenesis is required. A simple mechanism for scalegenesis is the Coleman-Weinberg mechanism. This is, however, not compatible with the observed masses of SM particles in order to generate the electroweak scale. Therefore, we need an extension of the SM for the use of the Coleman-Weinberg mechanism. We discuss details in Section 4.

Within low energy theories, however, we cannot explain why the SM or its extended models becomes scale invariant at a certain UV scale or the Planck scale. This is nothing but a strong assumption. It is interesting whether or not the scale invariant effective theory at the Planck scale is naturally defined from UV theories including quantum gravity. We will discuss this issue within the asymptotic safety scenario for quantum gravity in Section 5. In particular, we will see that a large anomalous dimension could be induced by graviton fluctuations above the Planck scale and thus it could provide natural solutions to the gauge hierarchy problem. In particular, classical scale invariance at the Planck scale emerges as a boundary condition from asymptotically safe quantum gravity.

\section{Scalegenesis}

If we impose classical scale symmetry on the SM, there is no corresponding scale to the electroweak one. Therefore, we need a mechanism to generate a scale. There are two ways for scalegenesis: the one is the Coleman-Weinberg mechanism [7] in the perturbation theory; and the other relies on the strong dynamics like quantum chromodynamics. The next section is devoted to discussing the basis mechanism of scalegenesis. We might think that the most minimal way to generate the electroweak scale is the Coleman-Weinberg mechanism within the SM. Unfortunately, this does not work since the observed SM particle masses do not satisfy the condition for scalegenesis. We see this fact in Section 4.3. Hence, we need an extension of the SM. In Section 4.5, as an example of scalegenesis, we introduce an extended model of the SM where a strongly interacting scalar-gauge theory in a hidden section is given.

\subsection{Condition for scalegenesis}

We review the Coleman-Weinberg mechanism bases on the original paper [7] to understand the crucial point for the generation of a scale. We consider a U(1) scalar-gauge theory whose action is given by

$$
S=\int \mathrm{d}^{4} x\left[\frac{1}{2}\left(D_{\mu} \phi\right)^{2}-\frac{\lambda}{4 !} \phi^{4}-\frac{1}{4} F_{\mu \nu} F^{\mu \nu}\right]
$$


where $\phi$ is a complex scalar field, $F_{\mu \nu}$ is the field strength of $A_{\mu}, D_{\mu}=\partial_{\mu}-i e A_{\mu}$ is the covariant derivative. The effective potential for the scalar field at the one-loop level is computed as

$$
V_{\mathrm{eff}}(\phi)=\frac{\lambda}{4 !} \phi^{4}+\left(\frac{5 \lambda^{2}}{18}+3 e^{4}\right) \frac{\phi^{4}}{64 \pi^{2}}\left(\ln \frac{\phi^{2}}{v_{\phi}^{2}}-\frac{25}{6}\right):=\frac{\lambda_{\mathrm{eff}}(\phi)}{4 !} \phi^{4},
$$

where $v_{\phi}=\langle\phi\rangle$. The vacuum condition is obtained by the first derivative of the effective potential with respect to $\phi$, namely

$$
\left.\phi \frac{\mathrm{d} V_{\mathrm{eff}}(\phi)}{\mathrm{d} \phi}\right|_{\phi=v_{\phi}}=\left.0 \quad \Longleftrightarrow \quad\left[4 \lambda_{\mathrm{eff}}(\phi)+\beta_{\lambda}\right]\right|_{\phi=v_{\phi}}=0
$$

where $\beta_{\lambda}=\phi \frac{\mathrm{d} \lambda_{\text {eff }}(\phi)}{\mathrm{d} \phi}$ in the beta function of the quartic coupling. From this we find, for a small $\lambda$,

$$
\left(\lambda-\frac{33 e^{4}}{8 \pi^{2}}\right) v_{\phi}=0
$$

We see from this that the requirement of a non-vanishing vacuum $v_{\phi} \neq 0$ gives a relation between coupling constants: $\lambda=33 e^{4} / 8 \pi^{2}$. Thus, one of two dimensionless couplings ( $\lambda$ and $e$ ) changes to the dimensionful quantity, i.e. $v_{\phi}$. Coleman and Weinberg called this mechanism the dimensional transmutation. Recently, the author and his collaborator have suggested calling it scalegenesis [26].

The crucial point for the generation of a scale in the Coleman-Weinberg mechanism is that the effective quartic coupling constant becomes negative for small field values. In this simple model, the gauge coupling plays a central role for this: In terms of the renormalization group equation, one has the loop effect of the gauge field on the beta function of the scalar quartic coupling such that $\beta_{\lambda} \supset+e^{4}$. That is, the effect of the gauge coupling makes the RG flow of the quartic coupling negative towards the IR (small field value) region. An other possible model of the Coleman-Weinberg mechanism is the system with the three scalar fields, e.g. the Higgs field plus two additional singletscalar fields [24]. The coupling constants $\lambda_{i j}$ between two different scalar fields such as the Higgs portal coupling contribute to the beta functions of quartic couplings $\lambda_{i}$ so that $\beta_{\lambda_{i}} \supset+\lambda_{i j}^{2}$ where indices denote species of scalar fields.

We here note that in the perturbative scalegenesis, the scalar mass parameter, which corresponds to the curvature at the origin of the effective potential, vanishes. This reflects the fact that the scale anomaly does not induce the soft breaking of scale symmetry as mentioned in the previous section. On the other hand, in scalegenesis due to non-perturbative dynamics, scale symmetry is spontaneously broken and the scalar field obtains a dynamical (constituent) mass $M^{2}$. In particular, we observe a divergence of the quartic coupling at a certain scale which is an origin of a scale. We see this fact in Section 4.5.

\subsection{Effective couplings}

An interesting question is how we can experimentally distinguish scalegensis by the ColemanWeinberg mechanism from the SM type due to a negative scalar mass parameter. Indeed, between them, there are large differences of the Higgs effective couplings which are defined as

$$
\lambda^{(2)}=\left.\frac{1}{2 v_{h}^{2}} \frac{\mathrm{d}^{2} V}{\mathrm{~d} h^{2}}\right|_{h=v_{h}}, \quad \lambda^{(3)}=\left.\frac{1}{6 v_{h}} \frac{\mathrm{d}^{3} V}{\mathrm{~d} h^{3}}\right|_{h=v_{h}}, \quad \lambda^{(4)}=\left.\frac{1}{6} \frac{\mathrm{d}^{4} V}{\mathrm{~d} h^{4}}\right|_{h=v_{h}} .
$$


Let us see explicitly these couplings for both the SM and the Coleman-Weinberg cases. In the SM case in which the Higgs potential with $v_{h}=\sqrt{m^{2} / \lambda}$ is given by

$$
V_{\mathrm{SM}}(h)=-\frac{m^{2}}{2} h^{2}+\frac{\lambda}{4} h^{4},
$$

the Higgs effective couplings (4.5) become $\lambda_{\mathrm{SM}}^{(2)}=\lambda_{\mathrm{SM}}^{(3)}=\lambda_{\mathrm{SM}}^{(4)}=\lambda$ which leads to

$$
\begin{array}{ll}
\frac{\lambda_{\mathrm{SM}}^{(3)}}{\lambda_{\mathrm{SM}}^{(2)}}=1, & \frac{\lambda_{\mathrm{SM}}^{(4)}}{\lambda_{\mathrm{SM}}^{(2)}}=1 .
\end{array}
$$

We next suppose that an extension of the SM realizes electroweak scalegenesis by the ColemanWeinberg mechanism in the Higgs sector and gives the following effective potential,

$$
V_{\mathrm{CW}}(h)=\frac{\lambda_{\text {eff }}(h)}{4} h^{4} .
$$

For this potential, we obtain the vacuum from Eq. (4.3) and find

$$
\begin{aligned}
& \lambda_{\mathrm{CW}}^{(2)}=\frac{1}{2}\left(\lambda_{\mathrm{eff}}^{\prime}+\frac{\lambda_{\mathrm{eff}}^{\prime \prime}}{4}\right) \\
& \lambda_{\mathrm{CW}}^{(3)}=\frac{5}{3} \lambda_{\mathrm{CW}}^{(2)}+\frac{\lambda_{\mathrm{eff}}^{\prime \prime}}{6}+\frac{\lambda_{\mathrm{eff}}^{\prime \prime \prime}}{24}, \\
& \lambda_{\mathrm{CW}}^{(4)}=\frac{11}{3} \lambda_{\mathrm{CW}}^{(2)}+\lambda_{\mathrm{eff}}^{\prime \prime}+\frac{5 \lambda_{\mathrm{eff}}^{\prime \prime \prime}}{12}+\frac{\lambda_{\mathrm{eff}}^{\prime \prime \prime \prime}}{24},
\end{aligned}
$$

where a prime denotes the derivative $h \frac{\mathrm{d}}{\mathrm{d} h}$. Then, neglecting the higher order terms in Eqs. (4.10) and (4.11), we find the ratios [25]

$$
\begin{array}{ll}
\frac{\lambda_{\mathrm{CW}}^{(3)}}{\lambda_{\mathrm{CW}}^{(2)}}=\frac{5}{3}, & \frac{\lambda_{\mathrm{CW}}^{(4)}}{\lambda_{\mathrm{CW}}^{(2)}}=\frac{11}{3} .
\end{array}
$$

We see that the Higgs effective couplings (4.12) in the Coleman-Weinberg potential drastically deviate from the prediction from the SM (4.7). The collider experiments such as the international linear collider in future will measure the Higgs effective coupling precisely and clarify the origin of electroweak scalegenesis.

\subsection{Coleman-Weinberg mechanism in the SM}

We briefly investigate at the Coleman-Weinberg potential in the SM and see that the electroweak scalegenesis actually does not take place. We have the one-loop effective potential in the $\mathrm{SM}$,

$$
V_{\mathrm{eff}}(h)=\frac{\lambda_{H}}{4} h^{4}+\sum_{\alpha} \frac{N_{\alpha} M_{\alpha}^{4}(h)}{64 \pi^{2}}\left(\ln \frac{M_{\alpha}^{2}(h)}{v_{h}^{2}}-C_{\alpha}\right)=: \frac{\lambda_{H, \mathrm{eff}}(h)}{4} h^{4},
$$

where $\alpha=(W, Z, t, h) ; N_{W}=6, N_{Z}=3, N_{t}=-12$ and $N_{h}=1$; and $C_{h, t}=3 / 2$ and $C_{W, Z}=5 / 6$; and the masses at the tree level are given by

$$
M_{W}^{2}=\frac{g_{2}^{2}}{4} h^{2}, \quad M_{Z}^{2}=\frac{g_{2}^{2}+g_{1}^{2}}{4} h^{2}, \quad M_{t}^{2}=\frac{y_{t}^{2}}{2} h^{2}, \quad M_{h}^{2}=3 \lambda_{H} h^{2} .
$$


Here, $\lambda_{H, \text { eff }}(h)$ is the effective quartic coupling as a functions of $h$. We have neglected contributions from other SM particles in the effective potential (4.13). The vacuum condition (4.3) in the present case yields

$$
v_{h}^{2}=\frac{-36 M_{t}^{4}+6 M_{W}^{4}+3 M_{Z}^{4}+3 M_{h}^{4}}{16 \pi^{2} M_{h}^{2}} .
$$

For the observed masses, $M_{W}=80 \mathrm{GeV}, M_{Z}=91 \mathrm{GeV}, M_{t}=171 \mathrm{GeV}$ and $M_{h}=125 \mathrm{GeV}$, the relation (4.15) yields a negative value of $v_{h}^{2}$. Therefore, even a finite value of the vacuum cannot be generated. This is because the Higgs quartic coupling does not become negative for small field values. If the top-quark mass was smaller or the Higgs and gauge boson masses were larger, a finite scale would be generated by the Coleman-Weinberg mechanism.

\subsection{Scale from new physics}

As we have seen in Section 4.3, the Coleman-Weinberg mechanism in the SM does not occur Therefore, we need an extension of the Higgs sector. The simplest extension may be an introduction of a scalar field $S$ coupled to the Higgs field through the Higgs-portal coupling. We give its Lagrangian as

$$
\mathscr{L}=\left.\mathscr{L}_{\mathrm{SM}}\right|_{m_{H} \rightarrow 0}-\lambda_{H S}\left(H^{\dagger} H\right)\left(S^{\dagger} S\right)+\left.\mathscr{L}_{S}\right|_{m_{S} \rightarrow 0},
$$

where $\left.\mathscr{L}_{S}\right|_{m_{S} \rightarrow 0}$ is the Lagrangian for the scalar field $S$ and we call it a hidden sector. We do not here specify the explicit form of $\left.\mathscr{L}_{S}\right|_{m_{S} \rightarrow 0}$. Assuming that the Coleman-Weinberg mechanism or the strong dynamics in the hidden sector works and then we obtain a non-trivial vacuum $\langle S\rangle \neq 0$ or $\left\langle S^{\dagger} S\right\rangle \neq 0$ which is proportional to a scale $\Lambda_{H}$ (smaller than the Planck scale), the Higgs-portal coupling plays a role of the Higgs mass term

$$
m_{H}^{2} \simeq \lambda_{H S} \Lambda_{H}^{2}
$$

In order for the Higgs field to obtain a non-trivial vacuum, the Higgs-portal coupling has to be negative. Moreover, $\Lambda_{H}$ has to be of order of about $\mathrm{TeV}$ scale if the Higgs-portal coupling is $\mathscr{O}\left(10^{-3}\right)-\mathscr{O}\left(10^{0}\right)$.

It seems, however, that the Higgs mass parameter in Eq. (4.17) receives a contribution from the quadratic divergence. Is it then irrelevant for the Higgs mass term by recalling the discussion in Section 3? Its answer is No. Indeed, the quadratic divergence in Eq. (4.17) is physical. In order to clarify this, we discuss the Wilsonian RG for a simple system with two scalar fields,

$$
\Gamma_{k}[\phi] \simeq \int \mathrm{d}^{4} x\left[\frac{1}{2}\left(\partial_{\mu} \phi_{i}\right)^{2}+\frac{1}{2} m_{i}^{2} \phi_{i}^{2}+\frac{1}{4 !} \lambda_{i} \phi_{i}^{4}+\frac{1}{8} \lambda_{i j} \phi_{i}^{2} \phi_{j}^{2}\right]
$$

where $i=1,2, i \neq j, \lambda_{12}=\lambda_{21}$ and we assume that $m_{1}<m_{2}$. Using the flow equation (2.18), we 
obtain the RG equations,

$$
\begin{aligned}
\partial_{t} \bar{m}_{i}^{2} & =-2 \bar{m}_{i}^{2}-\frac{\bar{\lambda}_{i}}{32 \pi^{2}} \ell_{1}^{4}\left(\bar{m}_{i}^{2}\right)-\frac{\bar{\lambda}_{i j}}{32 \pi^{2}} \ell_{1}^{4}\left(\bar{m}_{j}^{2}\right) \\
& \simeq-2 \bar{m}_{i}^{2}-\frac{\bar{\lambda}_{i}+\bar{\lambda}_{i j}}{32 \pi^{2}} \ell_{1}^{4}(0)+\frac{\bar{\lambda}_{i}}{16 \pi^{2}} \ell_{2}^{4}(0) \bar{m}_{i}^{2}+\frac{\bar{\lambda}_{i j}}{16 \pi^{2}} \ell_{2}^{4}(0) \bar{m}_{j}^{2} \\
\partial_{t} \bar{\lambda}_{i} & =-\frac{3 \bar{\lambda}_{i}^{2}}{16 \pi^{2}} \ell_{2}^{4}\left(\bar{m}_{i}^{2}\right)-\frac{3 \bar{\lambda}_{i j}^{2}}{16 \pi^{2}} \ell_{2}^{4}\left(\bar{m}_{j}^{2}\right) \\
\partial_{t} \bar{\lambda}_{i j} & =-\frac{\bar{\lambda}_{i j}^{2}}{8 \pi^{2}}\left[\ell_{0}^{4}\left(\bar{m}_{i}^{2}\right) \ell_{1}^{4}\left(\bar{m}_{j}^{2}\right)+\ell_{0}^{4}\left(\bar{m}_{j}^{2}\right) \ell_{1}^{4}\left(\bar{m}_{i}^{2}\right)\right]+\frac{\bar{\lambda}_{i j}}{16 \pi^{2}}\left[\bar{\lambda}_{i} \ell_{2}^{4}\left(\bar{m}_{i}^{2}\right)+\bar{\lambda}_{j} \ell_{2}^{4}\left(\bar{m}_{j}^{2}\right)\right],
\end{aligned}
$$

In the beta functions of the scalar masses (4.19), we expand the threshold functions into polynomials of $\bar{m}_{i}^{2}$ and take into account of order of $\bar{m}_{i}^{2}$ by assuming $\bar{m}_{i}^{2} \ll 1$. Here we analyze the RG flows around the Gaussian fixed point. To see a weak mixing effect between the two scalar fields, we take into account a linear order of $\bar{\lambda}_{i j}$ in Eq. (4.19). The linearized RG equations around the Gaussian fixed point $\left(\bar{m}_{i *}^{2}, \bar{\lambda}_{i *}\right)=(0,0)$ and $\bar{\lambda}_{i j} \ll 1$ are given by

$$
\partial_{t}\left(\begin{array}{c}
\bar{m}_{1}^{2} \\
\bar{m}_{2}^{2} \\
\bar{\lambda}_{1} \\
\bar{\lambda}_{2} \\
\bar{\lambda}_{12}
\end{array}\right) \simeq\left(\begin{array}{ccccc}
-2 & \frac{\bar{\lambda}_{12}}{16 \pi^{2}} & -\frac{a}{32 \pi^{2}} & 0 & -\frac{a}{32 \pi^{2}} \\
\bar{\lambda}_{12} & -2 & 0 & -\frac{a}{32 \pi^{2}} & -\frac{a}{32 \pi^{2}} \\
16 \pi^{2} & 0 & 0 & 0 \\
0 & 0 & 0 & 0 \\
0 & 0 & 0 & 0 & 0 \\
0 & 0 & 0 & 0 & 0
\end{array}\right)\left(\begin{array}{c}
\bar{m}_{1}^{2} \\
\bar{m}_{2}^{2} \\
\bar{\lambda}_{1} \\
\bar{\lambda}_{2} \\
\bar{\lambda}_{12}
\end{array}\right),
$$

where we have used the Litim-type cutoff (2.32) to have $\ell_{1}^{4}(0)=a$ and $\ell_{2}^{4}(0)=1$. The stability matrix can be diagonalized by the rotation matrix,

$$
V \simeq\left(\begin{array}{ccccc}
1 & -1 & -\frac{a}{64 \pi^{2}} & \frac{a}{64 \pi^{2}} \frac{\bar{\lambda}_{12}}{32 \pi^{2}} & -\frac{a}{64 \pi^{2}}\left(1-\frac{\lambda_{12}}{32 \pi^{2}}\right) \\
1 & 1 & \frac{a}{64 \pi^{2}} \frac{\bar{\lambda}_{12}}{32 \pi^{2}} & -\frac{a}{64 \pi^{2}} & -\frac{a}{64 \pi^{2}}\left(1-\frac{\lambda_{12}}{32 \pi^{2}}\right) \\
0 & 0 & 1 & 0 & 0 \\
0 & 0 & 0 & 1 & 0 \\
0 & 0 & 0 & 0 & 1
\end{array}\right),
$$

and has the eigenvalues, i.e. the critical exponents $\left(\theta_{m_{1}}, \theta_{m_{2}}, \theta_{\lambda_{1}}, \theta_{\lambda_{2}}, \theta_{\lambda_{12}}\right) \simeq(-2,-2,0,0,0)$. Then the RG equations for the scalar masses reads

$$
\begin{aligned}
& \partial_{t} \widetilde{m}_{1}^{2}=-2 \widetilde{m}_{1}^{2}+\frac{\bar{\lambda}_{12}}{16 \pi^{2}} \widetilde{m}_{2}^{2}, \\
& \partial_{t} \widetilde{m}_{2}^{2}=-2 \widetilde{m}_{2}^{2},
\end{aligned}
$$


where we have defined

$$
\begin{aligned}
& \widetilde{m}_{1}^{2}=\frac{\bar{m}_{1}^{2}}{2}+\frac{\bar{m}_{2}^{2}}{2}+\frac{a \bar{\lambda}_{1}}{128 \pi^{2}}\left(1+\frac{\bar{\lambda}_{12}}{32 \pi^{2}}\right)+\frac{a \bar{\lambda}_{2}}{128 \pi^{2}}\left(1+\frac{\lambda_{12}}{32 \pi^{2}}\right)+\frac{a \bar{\lambda}_{12}}{64 \pi^{2}}\left(1+\frac{\lambda_{12}}{32 \pi^{2}}\right), \\
& \widetilde{m}_{2}^{2}=-\frac{\bar{m}_{1}^{2}}{2}+\frac{\bar{m}_{2}^{2}}{2}-\frac{a \bar{\lambda}_{1}}{128 \pi^{2}}\left(1-\frac{\bar{\lambda}_{12}}{32 \pi^{2}}\right)+\frac{a \bar{\lambda}_{2}}{128 \pi^{2}}\left(1-\frac{\lambda_{12}}{32 \pi^{2}}\right) .
\end{aligned}
$$

In Eqs. (4.24) and (4.25), the quadratic divergent terms depending on the cutoff scheme are subtracted. Let us now solve the RG equations. The solution to Eq. (4.25) is easily found to be

$$
\widetilde{m}_{2}^{2}(k)=\widetilde{m}_{2,0}^{2}\left(\frac{k}{\Lambda_{2}}\right)^{-2}
$$

where $\Lambda_{2}$ is a scale at which the mass of $\phi_{2}$ is given, e.g. the expectation value of $\phi_{2}$, and $\widetilde{m}_{2,0}$ is a boundary value of $\widetilde{m}_{2}$ at $k=\Lambda_{2}$. The avoidance of the scale hierarchy in the $\phi_{2}$ sector requires $\widetilde{m}_{2,0} \simeq 1$. Substituting this solution into Eq. (4.24) and ignoring the running of $\bar{\lambda}_{12}$, we find

$$
\widetilde{m}_{1}^{2}(k)=\widetilde{m}_{1,0}^{2}\left(\frac{k}{\Lambda_{2}}\right)^{-2}+\frac{\bar{\lambda}_{12}}{16 \pi^{2}} \widetilde{m}_{2,0}^{2}\left(\frac{k}{\Lambda_{2}}\right)^{-2} \ln \left(\frac{k}{\Lambda_{2}}\right) .
$$

We see from this equation that even if $\widetilde{m}_{1,0}^{2}=0$ the scalar field $\phi_{1}$ obtains a finite dimensionful mass

$$
\widehat{m}_{1}^{2}\left(\Lambda_{1}\right)=\frac{\bar{\lambda}_{12}}{16 \pi^{2}} \widehat{m}_{2,0}^{2}\left(\Lambda_{2}\right) \ln \left(\frac{\Lambda_{1}}{\Lambda_{2}}\right) \simeq \bar{\lambda}_{12} \Lambda_{2}^{2}
$$

where $\Lambda_{1}<\Lambda_{2}$, we have defined $\widehat{m}_{1}^{2}\left(\Lambda_{1}\right)=\widetilde{m}_{1}^{2}\left(\Lambda_{1}\right) \Lambda_{1}^{2}$ and $\widehat{m}_{2}^{2}\left(\Lambda_{2}\right)=\widetilde{m}_{2}^{2}\left(\Lambda_{2}\right) \Lambda_{2}^{2} \simeq \Lambda_{2}^{2}$ and have assumed that $\ln \left(\Lambda_{1} / \Lambda_{2}\right)$ is of order one. This is just Eq. (4.17). We conclude that the quadratic divergence in Eq. (4.17) or Eq. (4.30) is not be subtracted by rotating the theory space and then is a physical object independent from the cutoff scheme.

\subsection{A model for scalegenesis}

We show one of examples for scalegenesis owing to the strong dynamics of the gauge interaction $[26,27]$. We introduce a scale invariant hidden sector in which a scalar field is coupled to the $\mathrm{SU}\left(N_{c}\right)$ gauge field. The scalar field is also coupled to the Higgs field via the Higgs-portal coupling. The total Lagrangian reads

$$
\mathscr{L}=\left.\mathscr{L}_{\mathrm{SM}}\right|_{m_{H}=0}+\mathscr{L}_{\text {hidden }}
$$

Here the Lagrangian for the hidden sector is given by

$$
\mathscr{L}_{\text {hidden }}=-\frac{1}{2} \operatorname{tr} F^{2}+\left(\left[D^{\mu} S_{i}\right]^{\dagger} D_{\mu} S_{i}\right)-\hat{\lambda}_{S}\left(S_{i}^{\dagger} S_{i}\right)\left(S_{j}^{\dagger} S_{j}\right)-\hat{\lambda}_{S}^{\prime}\left(S_{i}^{\dagger} S_{j}\right)\left(S_{j}^{\dagger} S_{i}\right)+\hat{\lambda}_{H S}\left(S_{i}^{\dagger} S_{i}\right) H^{\dagger} H,
$$

where $F=F^{a} \tau^{a}$ is the field strength of $\mathrm{SU}\left(N_{c}\right)$ gauge field $A_{\mu}^{a} ; \tau^{a}$ is the generator of $\mathrm{SU}\left(N_{c}\right)$ gauge transformation; $S_{i}$ and $H$ are the new scalar field and the Higgs doublet field, respectively; indices on the scalar field $S$ stand for the flavor indices; and $D_{\mu}=\partial_{\mu}-i g A_{\mu}^{a} t^{a}$ is the covariant derivative. 
This hidden Lagrangian (4.32) is invariant under scaler symmetry, the $\mathrm{SU}\left(N_{c}\right)$ gauge symmetry and the $\mathrm{U}\left(N_{f}\right)$ flavor symmetry. Note that $\hat{\lambda}_{H S}$ takes a positive value and the Higgs quartic interaction is included in $\left.\mathscr{L}_{\mathrm{SM}}\right|_{m_{H}=0}$.

We assume that the $\mathrm{SU}\left(N_{c}\right)$ gauge symmetry is not broken by the dynamics in the hidden sector, but scale symmetry is spontaneosely broken. Due to the strong dynamics of the gauge field in the low energy region the $\mathrm{SU}\left(N_{c}\right)$ invariant scalar bilinear condensate takes place such that

$$
\left\langle S_{i}^{\dagger} S_{j}\right\rangle=\left\langle\sum_{a=1}^{N_{c}} S_{i}^{a \dagger} S_{j}^{a}\right\rangle \propto \delta_{i j} .
$$

Thus, the Higgs portal coupling takes a form of the (negative) Higgs mass parameter:

$$
m_{H}^{2}=-\hat{\lambda}_{H S}\left\langle S_{i}^{\dagger} S_{i}\right\rangle
$$

As a consequence, the Higgs field has a non-trivial vacuum $v_{h}=\sqrt{m_{H}^{2} / \lambda_{H}}$.

We would like to see that the scalegenesis introduced above actually takes place. It is, however, quite difficult to analyze the vacuum structure in the original Lagrangian (4.31). Instead, we here attempt to formulate an effective model which describes the dynamical scale symmetry breaking, à $l a$, the Nambu-Jona-Lasinio model as a chiral effective model of quantum chromodynamics. However, scale symmetry is broken by not only the bilinear condensation (4.33), but also the scale anomaly. Nevertheless, the latter breaking effect is hard, namely the breaking of scale symmetry is due to higher dimensional operators as discussed in the previous section. Therefore, we could ignore anomalous breaking effects in low energy regions. Under this assumption, we employ the following effective Lagrangian to describe the spontaneous scale symmetry breaking [26]: ${ }^{6}$

$$
\mathscr{L}_{\text {eff }}=\left(\left[\partial^{\mu} S_{i}\right]^{\dagger} \partial_{\mu} S_{i}\right)-\lambda_{S}\left(S_{i}^{\dagger} S_{i}\right)\left(S_{j}^{\dagger} S_{j}\right)-\lambda_{S}^{\prime}\left(S_{i}^{\dagger} S_{j}\right)\left(S_{j}^{\dagger} S_{i}\right)+\lambda_{H S}\left(S_{i}^{\dagger} S_{i}\right) H^{\dagger} H-\lambda_{H}\left(H^{\dagger} H\right)^{2},
$$

where we include the Higgs quartic coupling. It is supposed in the effective Lagrangian (4.35) that quantum fluctuations of the gauge field $A_{\mu}$ were integrated out and then the scalar fields and the coupling constants are defined as effective ones.

Using the auxiliary field method in the path integral formalism or the normal ordering method in the operator formalism, we can obtain the mean-field approximated effective Lagrangian so that

$$
\mathscr{L}_{\mathrm{MFA}}=\left(\left[\partial^{\mu} S_{i}\right]^{\dagger} \partial_{\mu} S_{i}\right)-M^{2}\left(S_{i}^{\dagger} S_{i}\right)-\lambda_{H}\left(H^{\dagger} H\right)^{2}+N_{f}\left(N_{f} \lambda_{S}+\lambda_{S}^{\prime}\right) f^{2}+\frac{\lambda_{S}^{\prime}}{2}\left(\phi^{a}\right)^{2}-2 \lambda_{S}^{\prime} \phi^{a}\left(S_{i}^{\dagger} t_{i j}^{a} S_{j}\right),
$$

where $f=\left(S_{i}^{\dagger} S_{i}\right) / N_{f}$ and $\phi^{a}=2\left(S_{i}^{\dagger} t_{i j}^{a} S_{j}\right)$ are auxiliary fields with $t^{a}$ the generator of the flavor $\mathrm{SU}\left(N_{f}\right)$ transformation, and the "constituent" scalar mass is given by

$$
M^{2}=2\left(N_{f} \lambda_{S}+\lambda_{S}^{\prime}\right) f-\lambda_{H S} H^{\dagger} H .
$$

Assuming that the bilinear condensate (4.33) is invariant under the $\mathrm{U}\left(N_{f}\right)$ flavor transformation, we can choose a vacuum state $\langle f\rangle \neq 0$ and $\left\langle\phi^{a}\right\rangle=0$. Then, we can set to $\phi^{a}=0$ in Eq. (4.36)

\footnotetext{
${ }^{6}$ The confinement effect in terms of the Polyakov loop is discussed in Ref. [28].
} 
in order to derive the effective potential. The scalar field $S_{i}$ takes the bilinear form in the Lagrangian (4.36), so that we can integrate out it and obtain the effective potential,

$$
V_{\mathrm{MFA}}(\bar{S}, f, H)=M^{2}\left(\bar{S}_{i}^{\dagger} \bar{S}_{i}\right)+\lambda_{H}\left(H^{\dagger} H\right)^{2}-N_{f}\left(N_{f} \lambda_{S}+\lambda_{S}^{\prime}\right) f^{2}+\frac{N_{c} N_{f}}{32 \pi^{2}} M^{4} \ln \frac{M^{2}}{\Lambda_{H}^{2}}
$$

where $\bar{S}_{i}$ is the background field of the scalar field $S_{i}$, we have employed the dimensional regularization and the $\overline{\mathrm{MS}}$ scheme to subtract a UV divergence; and $\Lambda_{H}$ is a renormalization point at which the quantum effect vanishes for $M=\Lambda_{H}$.

To find the vacuum of the system, we evaluate the gap equations, i.e. the stationary conditions,

$$
0=\frac{\partial}{\partial \bar{S}_{i}^{a}} V_{\mathrm{MFA}}=\frac{\partial}{\partial f} V_{\mathrm{MFA}}=\frac{\partial}{\partial H_{l}} V_{\mathrm{MFA}} \quad(l=1,2),
$$

from which we find a minimum ${ }^{7}$

$$
\left\langle\bar{S}_{i}\right\rangle=0, \quad\langle f\rangle=f_{0}=\frac{2 \lambda_{H}}{G} \Lambda_{H}^{2} \exp \left(\frac{32 \pi^{2} \lambda_{H}}{N_{c} G}-\frac{1}{2}\right), \quad|\langle H\rangle|^{2}=\frac{v_{h}^{2}}{2}=\frac{N_{f} \lambda_{H S}}{2 \lambda_{H}}\langle f\rangle,
$$

where $G=4 N_{f} \lambda_{H} \lambda_{S}-N_{f} \lambda_{H S}^{2}+4 \lambda_{H} \lambda_{S}^{\prime}$. At this vacuum the constituent scalar mass and the Higgs mass are given by

$$
\left\langle M^{2}\right\rangle=M_{0}^{2}=\frac{G}{2 \lambda_{H}}\langle f\rangle, \quad M_{h}^{2}=|\langle H\rangle|^{2}\left(\frac{16 \lambda_{H}^{2}\left(N_{f} \lambda_{S}+\lambda_{S}^{\prime}\right)}{G}+\frac{N_{c} N_{f} \lambda_{H S}^{2}}{8 \pi^{2}}\right) \simeq 2 N_{f} \lambda_{H S}\langle f\rangle,
$$

where we assumed a small $\lambda_{H S}$ in the Higgs mass. We see that the dimensionful quantity $\Lambda_{H}$ is generated at the quantum level and becomes an origin of the electroweak scale and the Higgs mass.

Let us here the relation between the generation of the scale $\Lambda_{H}$ and the behavior of the quartic coupling $\lambda_{S}$ in the hidden sector. For simplicity, we consider the $N_{f}=1$ case and set $\lambda_{H S}=0$. We have the effective potential for $\chi:=2 \lambda_{S} f$,

$$
V_{\mathrm{MFA}}\left(\chi ; \lambda_{S}\right)=-\frac{1}{4 \lambda_{S}} \chi^{2}+\frac{N_{c}}{32 \pi^{2}} \chi^{2} \ln \frac{\chi}{\Lambda_{H}^{2}}
$$

From

$$
\frac{\partial^{2} V_{\mathrm{MFA}}}{\partial \chi^{2}}\left(e^{t} \chi ; \bar{\lambda}_{S}\right)=\frac{\partial^{2} V_{\mathrm{MFA}}}{\partial \chi^{2}}\left(\chi ; \lambda_{S}(t)\right)
$$

the RG flow of the quartic coupling $\lambda_{S}$ reads

$$
\lambda_{S}(t)=\frac{\bar{\lambda}_{S}}{1-\frac{N_{c} \bar{\lambda}_{S}}{8 \pi^{2}} t}
$$

where $t$ is a dimensionless scaling parameter and we defined $\lambda_{S}(t=0)=\bar{\lambda}_{S}>0$. Obviously, there is a Landau pole at $t=t_{c}=8 \pi^{2} / N_{c} \bar{\lambda}_{S}$. Since the present analysis does not rely on the

\footnotetext{
${ }^{7}$ In general, one can consider three possibilities as a vacuum solution: (i) $\left\langle\bar{S}_{i}^{a}\right\rangle \neq 0$ and $\langle f\rangle=0$; (ii) $\left\langle\bar{S}_{i}^{a}\right\rangle=0$ and $\langle f\rangle=0$; (iii) $\left\langle\bar{S}_{i}^{a}\right\rangle=0$ and $\langle f\rangle \neq 0$. The last condition (iii) yields absolute minimum of the effective potential.
} 
perturbative expansion of $\lambda_{S}$, the system is still defined for $t>t_{c}$ for which the effective quartic coupling becomes negative. The minimum of the effective potential (4.42) is located at

$$
\chi_{\mathrm{m}}:=\Lambda_{H}^{2} \exp \left(\frac{8 \pi^{2}}{N_{\mathrm{c}} \bar{\lambda}_{S}}-\frac{1}{2}\right)
$$

The corresponding dimensionless scaling parameter to this minimum is

$$
t=t_{\mathrm{m}}:=\ln \left(\frac{\chi_{\mathrm{m}}}{\mu^{2}}\right)=\left(\frac{8 \pi^{2}}{N_{c} \bar{\lambda}_{S}}-\frac{1}{2}\right)+\ln \frac{\Lambda_{H}^{2}}{\mu^{2}}=t_{c}-\frac{1}{2}+\ln \frac{\Lambda_{H}^{2}}{\mu^{2}},
$$

where $\mu$ is a dimensionful scale at which $\bar{\lambda}_{S}$ is given. In particular, for the choice ${ }^{8} \mu=\Lambda_{H} e^{-1 / 4}$, we have $t_{\mathrm{m}}=t_{c}$. We see that the non-trivial vacuum (4.45) is related to the Landau pole of the effective quartic coupling $\lambda_{S}(t)$.

We note here that for $\chi \rightarrow \infty$ the quadratic coupling goes to zero from the negative side of $\lambda_{S}(t)$, i.e. it is asymptotically free. In such a case, however, the potential of the scalar field $S$ is unbounded, so that $S$ is unstable. This situation could be improved by taking account the dynamics of the gauge field. We will discuss this issue elsewhere.

We finally comment on several phenomenological implications from this model. The composite scalars $\phi^{a}$ are stable due to the flavor symmetry, so that they can be dark matter candidates. The prediction for the spin-independent elastic cross section of $\phi^{a}$ off the nucleon could be tested by the direct detection experiments [26, 29]. In this model, the scale and electroweak phase transitions take place at finite temperature. In particular, the scale phase transition becomes the strong firstorder, i.e. $\langle f\rangle^{1 / 2} / T_{c} \gtrsim 1$ where $T_{c}$ is the critical temperature [30]. The strong first-order scale phase transition produces gravitational waves which could be observed by the future space gravitational wave antennas [31].

\section{Asymptotically safe quantum gravity and the gauge hierarchy problem}

We now consider the high energy physics above the Planck scale, especially asymptotically safe quantum gravity which is formulated as a nonperturbatively renormalizable quantum field theory $[32,33,34]$. It is well-known that quantum gravity based on the Einstein-Hilbert action is not perturbatively renormalizable. In other words, the Newton constant, which has canonical massdimension -2 , is irrelevant at the Gaussian fixed point. On the other hand, it is essential for the asymptotic safety scenario that gravitational couplings has a non-trivial UV fixed point at which the Newton constant is relevant. A numerous work using the Wilsonian RG has shown evidences of the existence of such a fixed point. See recent reviews [35, 36, 37, 38, 39].

We employ the Einstein-Hilbert truncation for the effective action,

$$
\Gamma_{k}^{\mathrm{EH}}=\int \mathrm{d}^{4} x \sqrt{g}\left[\Lambda_{\mathrm{CC}}-\frac{1}{16 \pi G} R\right]+\Gamma_{\mathrm{gh}}+\Gamma_{\mathrm{gf}},
$$

\footnotetext{
${ }^{8}$ This choice of $\mu$ is equivalent to the redefinition of $\lambda_{S}(t=0)=\bar{\lambda}_{S}$ with the choice $\mu=\Lambda_{H}$ such that $\frac{8 \pi^{2}}{N_{\mathrm{c}} \bar{\lambda}_{S}}-\frac{1}{2} \rightarrow$ $\frac{8 \pi^{2}}{N_{\mathrm{c}} \bar{\lambda}_{S}}$ in Eq. (4.45). This is nothing but the dimensional transmutation or scalegensis: the degree of freedom of the dimensionless coupling $\bar{\lambda}_{S}$ changes to that of the dimensionful parameter.
} 


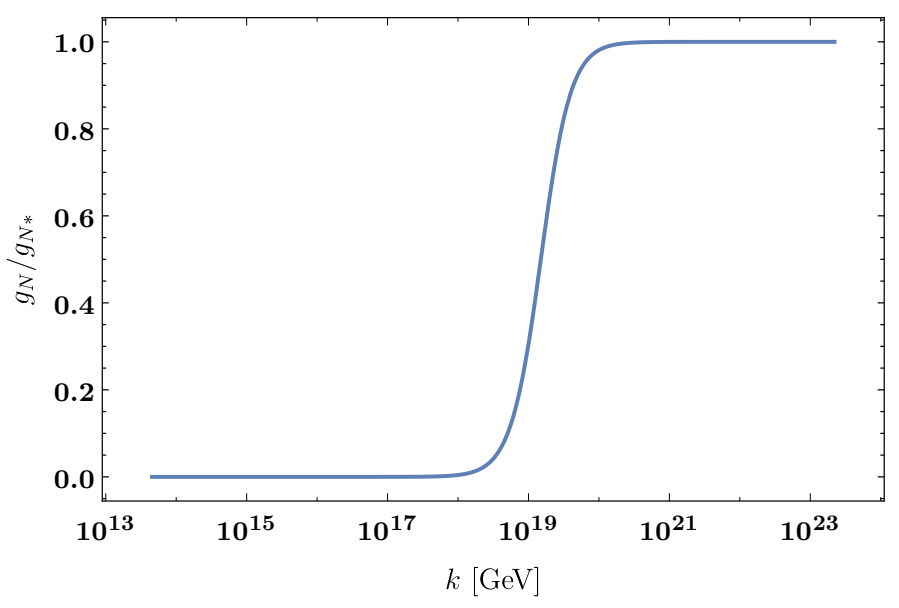

Figure 3: Schematic figure for the RG flow of the dimensionless Newton constant.

where $\Lambda_{\mathrm{CC}}$ is the cosmological constant; $G$ is the dimensionful Newton constant; $\sqrt{g}$ is the determinant of metric; $R$ is the Ricci scalar; and $\Gamma_{\mathrm{gh}}$ and $\Gamma_{\mathrm{gf}}$ are the ghost and gauge fixing actions for diffeomorphism, respectively. The RG equation for the Newton constant is given by

$$
\partial_{t} g_{N}=\left(2+\eta_{g}\right) g_{N}
$$

where $g_{N}=G k^{2}$ is the dimensionless Newton constant. Here, $\eta_{g}$ is the anomalous dimension induced by graviton fluctuations and has been computed. See e.g. Refs. [40, 41, 42, 43, 44]. The important fact is that $\eta_{g}$ takes a negative value smaller than -2 at the UV fixed point. Therefore, the Newton constant becomes a relevant parameter at the UV fixed point, whereas we observe the irrelevant Newton constant with the critical exponent $\theta_{g_{N}}=-2\left(\eta_{g}=0\right)$ at the Gaussian fixed point, $g_{N *}=0$. In Fig. 3, we plot a schematic figure of the RG running of the dimensionless Newton constant with neglecting the cosmological constant.

One of advantages of asymptotically safe gravity is a strong predictability to the low energy dynamics of particles. Indeed, the Higgs mass of a mass of $125 \mathrm{GeV}$ with a few $\mathrm{GeV}$ uncertainty was predicted before the discovery of the Higgs boson at the LHC [45]. Besides, physical quantities such as quark masses could be predicted within the asymptotic safety program; see [46, 47, 48, $49,50]$. It is crucial for these predictions how the anomalous dimensions induced by graviton fluctuations contribute to the beta functions of matter couplings.

Let us here investigate quantum gravity effects on the scalar potential. To this end, we replace the cosmological constant to the effective potential of a $O(N)$-scalar field $\phi$, i.e. $\Lambda_{\mathrm{CC}} \rightarrow U(\phi)$, and add the kinetic term of the scalar field. The RG equation for the effective potential taking into account only the quantum gravity effects reads [43]

$$
\partial_{t} \tilde{U}=-4 \tilde{U}+\tilde{\phi} \tilde{U}^{\prime}+\frac{1}{24 \pi^{2}}\left[\frac{5}{1-v}+\frac{1}{1-v / 4}-3\right],
$$

where we define dimensionless quantities, $\tilde{\phi}=\phi / k, \tilde{U}(\tilde{\phi})=U(\phi) / k^{4}$ and $v=16 \pi g_{N} \tilde{U}$, and the prime denotes the derivative with respect to $\tilde{\phi}$. The first two terms on the right-hand side of Eq. (5.3) are the canonical scaling of the effective potential, while the third term is the graviton loop effect. 
Expanding the effective potential into a polynomial of $\tilde{\phi}^{2}$, we obtain the RG equation for the scalar mass term,

$$
\partial_{t} \bar{m}^{2}=\beta_{m}=\left(-2+\gamma_{m}^{g}\right) \bar{m}^{2},
$$

with the anomalous dimension induced by graviton fluctuations,

$$
\gamma_{m}^{g}=\frac{g_{N}}{6 \pi}\left[\frac{20}{\left(1-v_{0}\right)^{2}}+\frac{1}{\left(1-v_{0} / 4\right)^{2}}\right]
$$

where $v_{0}=16 \pi g_{N} \tilde{U}(0)$ with $\tilde{U}(0)=\Lambda_{\mathrm{CC}} / k^{4}$ the dimensionless cosmological constant. From the RG equation (5.4) we find the critical exponent of the scalar mass term above the Planck scale,

$$
\theta_{m} \simeq-\left.\frac{\partial \beta_{m}}{\partial \bar{m}^{2}}\right|_{\substack{g_{N}=g_{N *} \\ v_{0}=v_{0 *}}}=2-\frac{g_{N *}}{6 \pi}\left[\frac{20}{\left(1-v_{0 *}\right)^{2}}+\frac{1}{\left(1-v_{0 *} / 4\right)^{2}}\right] .
$$

For a finite value of $g_{N *}$, the anomalous dimension becomes finite, so that the energy scaling of the scalar mass parameter is different from the canonical one.

We here suppose that $\gamma_{m}^{g}>2$. In this case, the sign of the critical exponent of the scalar mass becomes negative, namely the scalar mass parameter is irrelevant. This situation could provide possible solutions to the gauge hierarchy problem: One of them is the resurgence mechanism [51] in which the scalar mass parameter behaves as the blue solid lien in Fig. 4. The scalar mass parameter shrinks towards zero in the regions above the Planck scale and then increases such that $m_{H}^{2} / v_{h}^{2} \simeq 0.2$ at the electroweak scale due to the decoupling of quantum gravity effects below the Planck scale. Hence, the Higgs mass parameter is tuned so as to be $m_{0}^{2} / M_{\mathrm{pl}}^{2} \simeq 10^{36}$ around the Planck scale. This is called as the self-tuned criticality [18]. The scalar mass parameter increases towards UV scales and then diverges. In order for the scalar mass (or equivalently the electroweak scale) to be UV safe and predictable (irrelevant), one has to have a quite small fixed point $\bar{m}_{*}^{2} \simeq 10^{-36}$. So far, we have not found such a fixed point within simple truncated systems. Instead, we find the Gaussian fixed point $\bar{m}_{*}^{2}=0$ for which the RG flow of the scalar mass keeps zero. That is, the asymptotically safe condition in the continuum limit $k \rightarrow \infty$ implies that the low energy effective theory at the Planck scale could be (classically) scale invariant.

We now give several comments in order. The magnitude of the anomalous dimension $\gamma_{m}^{g}$ depends on the value of the fixed point of gravitational couplings, so that we need more precise analyze in order to establish the scenarios discussed above. In the scenarios with a negative critical exponent of the scalar mass parameter, the quadratic divergence is irrelevant: The UV theory is characterized by the UV fixed point and the low energy effective theory is given as a deviation from the fixed point. That is, the quadratic divergence is subtracted. However, the gauge hierarchy problem arises if there is a large intermediate scale between the Planck scale and the electroweak scale, e.g. the grand unification scale, $\Lambda_{\mathrm{GUT}}$. The large intermediate scale quadratically contributes to the Higgs mass parameter as discussed in Section 4.4.

\section{Summary}

We have revisited the gauge hierarchy problem in elementary particle physics. The quadratic divergence appearing in the scalar mass parameter may be spurious when we discuss the low energy 


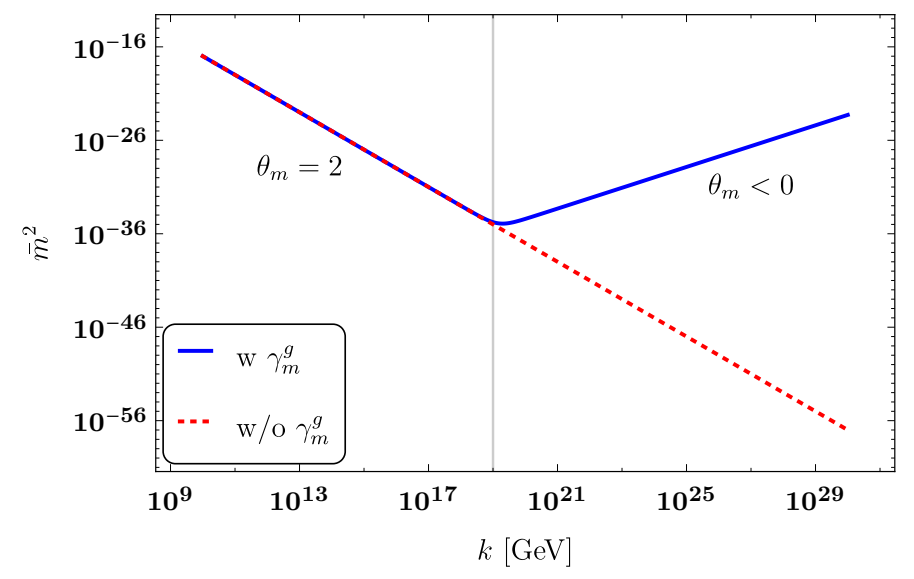

Figure 4: Schematic figure for the RG flow of the scalar mass term with and without $\gamma_{m}^{g}>2$.

dynamics of particles. The other aspect of the gauge hierarchy problem arises from the fact that the dimensionless scalar mass parameter $\bar{m}^{2}$ is a relevant parameter with the critical exponent 2, namely behaves as $\bar{m}^{2} \sim k^{-2}$. This fact with $m_{H}^{2}\left(v_{h}\right) / v_{h}^{2} \simeq 0.1$ implies that the Higgs mass at the Planck scale is quite smaller than the Planck scale: $m_{H}^{2}\left(M_{\mathrm{pl}}\right) / M_{\mathrm{pl}} \simeq 10^{-36}$. A possible solution within the low energy physics is the imposition of classical scale invariance on the bare action. Then, we extend the SM such that a scale $\Lambda_{H}$, which induces the electroweak scale, is generated. In this paper, we have discussed such an extension of the SM based on the strongly interacting scalargauge theory in a hidden sector. A newly added scalar field $S$ is coupled to the Higgs field $H$ via the Higgs-portal coupling $\lambda_{H S}\left(H^{\dagger} H\right)\left(S^{\dagger} S\right)$ which changes to the Higgs mass parameter proportional to the quadratic scale, i.e. $m_{H}^{2} \sim \lambda_{H S} \Lambda^{2}$. This quadratic divergence is physical, namely independent from the choice of the regularization scheme.

Although classical scale invariance provides a direction of an extension of the SM, it is a strong assumption. To establish the scenario for electroweak scalegenesis, we have to discuss the high energy physics including quantum gravity beyond the Planck scale. In this paper, we have introduced asymptotically safe quantum gravity which is realized as a non-perturbative field theory. The anomalous dimensions induced by graviton fluctuations could drastically change the energy scaling of coupling constants above the Planck scale. In particular, the graviton anomalous dimension $\gamma_{m}^{g}$ tends to make the critical exponent of the scalar mass parameter smaller than its canonical dimension. If $\gamma_{m}^{g}$ is larger than 2 , the scalar mass parameter becomes irrelevant. In such a case, potential solutions to the gauge hierarchy problem are given: One of them is the resurgence mechanism in which the small Higgs mass parameter $\bar{m}_{H}^{2} / M_{\mathrm{pl}} \simeq 10^{-36}$ is self-organized by quantum gravity effects. The other is classical scale invariance. That is, scale invariance at the Planck scale could be naturally realized as a consequence of the irrelevance of the scalar mass parameter above the Planck scale. The gauge hierarchy problem may provide hints for a connection between low and high energy physics.

\section{Acknowledgement}

The author thanks J. Kubo and C. Wetterich for collaborations and discussions. He is supported by the Alexander von Humboldt foundation. 


\section{References}

[1] E. Gildener, Phys. Rev. D 14, 1667 (1976).

[2] S. Weinberg, Phys. Lett. 82B, 387 (1979).

[3] G. Aad et al. [ATLAS Collaboration], Phys. Lett. B 716, 1 (2012)

[4] S. Chatrchyan et al. [CMS Collaboration], Phys. Lett. B 716, 30 (2012)

[5] C. Wetterich, Phys. Lett. 140B, 215 (1984).

[6] W. A. Bardeen, FERMILAB-CONF-95-391-T.

[7] S. R. Coleman and E. J. Weinberg, Phys. Rev. D 7, 1888 (1973). doi:10.1103/PhysRevD.7.1888

[8] C. Wetterich, Phys. Lett. B 301, 90 (1993)

[9] J. Berges, N. Tetradis and C. Wetterich, Phys. Rept. 363, 223 (2002)

[10] D. F. Litim, Phys. Rev. D 64, 105007 (2001)

[11] K. Gawedzki and A. Kupiainen, Nucl. Phys. B 257, 474 (1985).

[12] H. Aoki and S. Iso, Phys. Rev. D 86, 013001 (2012)

[13] J. I. Sumi, W. Souma, K. I. Aoki, H. Terao and K. Morikawa, hep-th/0002231.

[14] Y. Hamada, H. Kawai and K. y. Oda, Phys. Rev. D 87, no. 5, 053009 (2013) Erratum: [Phys. Rev. D 89, no. 5, 059901 (2014)]

[15] S. P. Martin, Adv. Ser. Direct. High Energy Phys. 21, 1 (2010) [Adv. Ser. Direct. High Energy Phys. 18, 1 (1998)]

[16] M. J. G. Veltman, Acta Phys. Polon. B 12, 437 (1981).

[17] C. Wetterich, Phys. Lett. 104B, 269 (1981).

[18] S. Bornholdt and C. Wetterich, Phys. Lett. B 282, 399 (1992).

[19] C. Wetterich, Phys. Lett. B 718, 573 (2012)

[20] H. Terao and A. Tsuchiya, arXiv:0704.3659 [hep-ph].

[21] K. Symanzik, Commun. Math. Phys. 18, 227 (1970).

[22] C. G. Callan, Jr., Phys. Rev. D 2, 1541 (1970).

[23] J. H. Lowenstein and W. Zimmermann, Commun. Math. Phys. 46, 105 (1976).

[24] J. Haruna and H. Kawai, PTEP 2020, no. 3, 033B01 (2020)

[25] D. Chway, T. H. Jung, H. D. Kim and R. Dermisek, Phys. Rev. Lett. 113, no. 5, 051801 (2014)

[26] J. Kubo and M. Yamada, Phys. Rev. D 93, no. 7, 075016 (2016)

[27] J. Kubo, K. S. Lim and M. Lindner, Phys. Rev. Lett. 113, 091604 (2014)

[28] J. Kubo and M. Yamada, JHEP 1810, 003 (2018)

[29] J. Kubo, Q. M. B. Soesanto and M. Yamada, Eur. Phys. J. C 78, no. 3, 218 (2018)

[30] J. Kubo and M. Yamada, PTEP 2015, no. 9, 093B01 (2015)

[31] J. Kubo and M. Yamada, JCAP 1612, 001 (2016) 
[32] S. Weinberg, Chap. 16 in General Relativity ed. by Hawking, S.W. and Israel, W (1979).

[33] M. Reuter, Phys. Rev. D 57, 971 (1998)

[34] W. Souma, Prog. Theor. Phys. 102, 181 (1999)

[35] A. Eichhorn, Found. Phys. 48, no. 10, 1407 (2018)

[36] R. Percacci, “An Introduction to Covariant Quantum Gravity and Asymptotic Safety,”

[37] A. Eichhorn, Front. Astron. Space Sci. 5, 47 (2019)

[38] M. Reuter and F. Saueressig, "Quantum Gravity and the Functional Renormalization Group : The Road towards Asymptotic Safety,"

[39] C. Wetterich, arXiv:1901.04741 [hep-th].

[40] K. y. Oda and M. Yamada, Class. Quant. Grav. 33, no. 12, 125011 (2016)

[41] Y. Hamada and M. Yamada, JHEP 1708, 070 (2017)

[42] A. Eichhorn, Y. Hamada, J. Lumma and M. Yamada, Phys. Rev. D 97, no. 8, 086004 (2018)

[43] J. M. Pawlowski, M. Reichert, C. Wetterich and M. Yamada, Phys. Rev. D 99, no. 8, 086010 (2019)

[44] C. Wetterich and M. Yamada, Phys. Rev. D 100, no. 6, 066017 (2019)

[45] M. Shaposhnikov and C. Wetterich, Phys. Lett. B 683, 196 (2010)

[46] A. Eichhorn and A. Held, Phys. Lett. B 777, 217 (2018)

[47] A. Eichhorn, A. Held and C. Wetterich, Phys. Lett. B 782, 198 (2018)

[48] A. Eichhorn and A. Held, Phys. Rev. Lett. 121, no. 15, 151302 (2018)

[49] A. Eichhorn, A. Held and C. Wetterich, arXiv:1909.07318 [hep-th].

[50] R. Alkofer, A. Eichhorn, A. Held, C. M. Nieto, R. Percacci and M. Schröfl, arXiv:2003.08401 [hep-ph].

[51] C. Wetterich and M. Yamada, Phys. Lett. B 770, 268 (2017) 